Check for updates

Cite this: Phys. Chem. Chem. Phys., 2022, 24, 2758

Received 30th November 2021, Accepted 12th January 2022

DOI: $10.1039 / \mathrm{d} 1 \mathrm{cp} 05468 f$

rsc.li/pccp

\section{Detecting chirality in mixtures using nanosecond photoelectron circular dichroism}

\author{
Simon T. Ranecky, ${ }^{a}$ G. Barratt Park, (D) bcd Peter C. Samartzis, (D) ${ }^{e}$ \\ loannis C. Giannakidis, (D) ${ }^{\text {ef }}$ Dirk Schwarzer, (D) ${ }^{c}$ Arne Senftleben, (D) ${ }^{a}$ \\ Thomas Baumert (D) ${ }^{a}$ and Tim Schäfer (D) *bc
}

\begin{abstract}
We report chirality detection of structural isomers in a gas phase mixture using nanosecond photoelectron circular dichroism (PECD). Combining pulsed molecular beams with high-resolution resonance enhanced multi-photon ionization (REMPI) allows specific isolated transitions belonging to distinct components in the mixture to be targeted.
\end{abstract}

Identification of molecules in mixtures is commonly performed using well-established gas chromatographic based techniques often coupled with mass spectrometry (GC-MS). ${ }^{1}$ With this technique, differentiation of enantiomers of chiral molecules is demanding as MS provides no enantiosensitivity when using classical ionization techniques and enantiosensitive MS using circular dichroism is still a field of research. ${ }^{2-4}$ This lack of chiral sensitivity at the mass spectrometric part of GC-MS is usually compensated using sophisticated gas chromatographic approaches. For instance, multidimensional gas chromatography offers enantiosensitive detection employing chiral stationary phases. $^{5-7}$ Other MS-based techniques involving chiral recognition involve demanding enantioselective separation prior to ionization as employed in the kinetic method, hostguest diastereometric adduct formation, ion-molecule reactions, collision-induced dissociation of diastereometric adducts, and ion mobility spectrometry. ${ }^{8}$ Hence, detection methods that are directly sensitive to the molecular chirality, and allow for real-time and in situ measurements provide an advantageous and complementary alternative to classical enantiosensitive GC-MS approaches.

\footnotetext{
${ }^{a}$ Universität Kassel, Heinrich-Plett-Str. 40, Kassel 34132, Germany

${ }^{b}$ Georg-August-Universität Göttingen, Tammannstr. 6, Göttingen 37077, Germany.

E-mail: tschaef4@gwdg.de

${ }^{c}$ Max Planck Institut für Biophysikalische Chemie, Am Fassberg 11, Göttingen 37077, Germany

${ }^{d}$ Department of Chemistry and Biochemistry, Texas Tech University, Box 41061, Lubbock, TX 79409-1061, USA

${ }^{e}$ Institute of Electronic Structure and Lasers, Foundation for Research and Technology - Hellas (FORTH), P. O. Box 1527, Heraklion 71110, Greece

${ }^{f}$ Department of Materials Science and Technology, University of Crete, Heraklion 71003, Greece
}

An example of a successful method for enantiomer-specific detection of chiral molecules in the gas phase is based on microwave spectroscopy. ${ }^{9,10}$ Here, an enantiomer-sensitive free induction decay (FID) is obtained by employing a three wave mixing scheme on rotational transitions. The FID's phase depends on the enantiomeric form of the molecule (Microwave three-wave mixing, M3WM). This method has opened new possibilities in chiral analysis and has already been successfully applied to mixtures of chiral molecules. ${ }^{11}$ Here, the detection scheme is intrinsically sensitive to the target molecule due to the rotational transitions and molecular discrimination in mixtures is possible.

An alternative approach for enantiosensitive detection in the gas phase is based on photoelectron circular dichroism (PECD): molecules are ionized using circularly polarized light and angular distributions of emitted photoelectrons are detected. The photoelectron emission angle depends on the combination of molecular handedness and photon helicity. ${ }^{12-14}$ The PECD, which is characteristic for each molecule, can be deduced from the photoelectron angular distribution (PAD). Ionization is performed by single photon ionization using radiation from synchrotrons as described in several review articles, ${ }^{15-18}$ or by resonant multi-photon ionization (REMPI) employing table-top femtosecond lasers. ${ }^{19-21}$ Recently, initial experiments on PECD have been performed using high-resolution nanosecond and picosecond laser systems. ${ }^{2-25}$ The PECD depends on several experimental parameters like wavelength and photoelectron kinetic energy, ${ }^{26-29}$ but once calibrated, it can be used as analytic tool capable of determining enantiomeric excess with sub $1 \%$ sensitivity. ${ }^{30,31}$

Recently, this method has been applied to identify the chirality of molecules in multi-component mixtures employing electron-ion coincidence measurements. ${ }^{32}$ Here, photoions are detected using femtosecond laser-based mass spectrometric time-of-flight techniques. Simultaneously, PADs are measured in coincidence with the ion mass, so that PECD values can be assigned to respective masses. ${ }^{21,33}$ Also recently, Comby et al. successfully measured in continuous photoelectron elliptical 
dichroism (c-PEELD) experiments the enantiomeric excess of fenchone within a fenchone/camphor mixture with an accuracy of $0.4 \%$ on a $10 \mathrm{~min}$ timescale and $5 \%$ accuracy with a temporal resolution of $3 \mathrm{~s}$. In their measurements, they record the molecular signatures of 3D photelectron angular distribution as a function of the laser ellipticity. With this approach, the authors were able to distinguish two components and determine enantiomeric excesses independent of the molecular mass. $^{34}$

In this proof-of-concept study, we present another alternative approach for enantiosensitive molecular identification in gas phase mixtures using nanosecond photoelectron circular dichroism based on our previous experiments on pure samples. ${ }^{23}$ We tune a high-resolution ns laser to moleculespecific resonances in order to selectively photoionize specific components in a supersonic molecular beam expansion containing a mixture of the structural isomers fenchone and camphor. This technique discriminates molecules by their REMPI transitions, and does not rely on different molecular masses. Hence, it provides a straightforward means of chirality detection in mixtures of structural isomers and conformers.

We performed the experiments on two different experimental set-ups. First, high-resolution REMPI spectra of fenchone/camphor mixtures were recorded in a pulsed molecular beam apparatus, which is explained in detail elsewhere. ${ }^{35,36}$ Briefly, we expand $(S)-(+)$-fenchone, (Sigma-Aldrich), and $(S)$ (-)-camphor (Sigma-Aldrich), and a well-defined mixture of both compounds in a pulsed molecular beam seeded in helium. Fenchone and camphor are transferred to the gas phase in a resistively heated reservoir, which is located directly upstream from a home-built nozzle. ${ }^{37}$ We have temperature control over different sections of the reservoir, ensuring that compounds with a lower vapor pressure experience higher temperatures for similar number densities in the gas phase. We resonantly ionize fenchone and camphor via the $3 \mathrm{~s}$ Rydberg state in a $2+1$ REMPI process at wavelength around $410 \mathrm{~nm}$ using the $1.5 \mathrm{~mJ}$ output of a frequency doubled dye laser (LIOPTEC, LiopStar, laser dye: Styryl 9) pumped by a frequency doubled Nd:YAG laser (Continuum, PowerLite $8010,532 \mathrm{~nm}, 10 \mathrm{~Hz}$ ). We produce ions by focusing through a $f=300 \mathrm{~mm}$ lens and accelerate them onto microchannel plates in chevron configuration (Topag, MCP 56-15) connected to a phosphor screen (ProxiVision). We record the total ion signal summed over all mass fragments as function of the wavelength to obtain REMPI spectra.

Angular distributions of emitted photoelectrons are obtained with a slightly different experimental setup. Using the same nozzle, we employ a photoelectron velocity map imaging (VMI) spectrometer, which has been explained in previous work. ${ }^{38}$ Laser setup, as well as photoelectron detection, is identical to our previous work on pure samples. ${ }^{23} \mathrm{We}$ record PADs for left circularly polarized light (LCP) and right circularly polarized light (RCP) using an achromatic quarterwaveplate (B. Halle, $300-470 \mathrm{~nm}$ achromatic with air gap). The quality of polarization is checked using a Glan-Laser polarizer (Thorlabs GL10) to determine the Stokes $\left|S_{3}\right|$ parameter, which

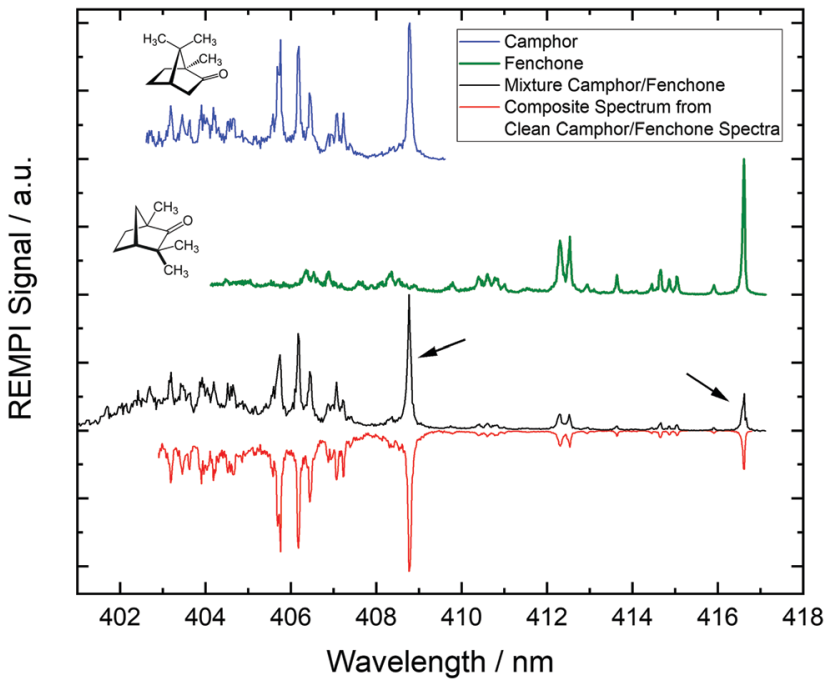

Fig. $12+1$ REMPI spectra of camphor (blue), fenchone (green), and a camphor/fenchone mixture (black) ionized via the $3 \mathrm{~s}$ Rydberg state using a Nd:YAG pumped nanosecond dye laser. We can reproduce the REMPI spectrum of the mixture by a linear combination of the REMPI spectra of clean camphor and fenchone (red). The black arrows indicate the $0_{0}^{0}$ transitions, at which we record PECD. They are well separated and shifted by ca. $8 \mathrm{~nm}$.

is above $96 \%$ for all measurements. At each chosen wavelength, we average for 1800 laser pulses ( $c a .3 \mathrm{~min}$ ) to record PAD after LCP and RCP ionization. PECD images are constructed by normalizing the PADs to one followed by subtraction of the RCP PAD from the LCP PAD and subsequent antisymmetrization. Additionally, three dimensional photoelectron distributions are reconstructed using an Abel inversion routine based on the pBasex algorithm. ${ }^{39}$ From the odd-order coefficients we calculate the linear PECD normalized to the total signal $c_{0}:{ }^{20}$

$$
\mathrm{LPECD}=\frac{1}{c_{0}}\left(2 c_{1}-\frac{1}{2} c_{3}+\frac{1}{4} c_{5}\right)
$$

We calculate the LPECD as a weighted average over the range of radii contained within the full width at half maximum (FWHM) of the photoelectron peak signal.

In Fig. 1 we show REMPI spectra of camphor, fenchone and of a camphor/fenchone mixture. The mixture is prepared by filling the reservoir with $c a .2 \mathrm{~g}$ of camphor and adding $0.1 \mathrm{ml}$ of fenchone without mixing solid and liquid. The temperature gradient across the reservoir is controlled such that the region containing fenchone is heated to $330 \mathrm{~K}$ and the region containing camphor is heated to $370 \mathrm{~K}$, ensuring similar contributions to the spectrum. Due to different structures, the $0_{0}^{0}$ transitions of the two isomers are shifted relative to one another by $c a$. $8 \mathrm{~nm}(114 \mathrm{meV})$. The spectra are well resolved as the cooling of internal degrees of freedom during the supersonic expansion leads to low rotational and vibrational temperatures.

We reproduce the spectrum of the mixture by a linear combination of the pure camphor spectrum and the pure fenchone spectrum as shown by the red line in Fig. 1 . The $0_{0}^{0}$ transition of fenchone is located at a spectral region in which 
no REMPI signal of camphor occurs. The spectral contribution of fenchone to the $0_{0}^{0}$ line of camphor in the spectrum of the mixture is deduced from the composite spectrum and is smaller than $1.5 \%$ for the experimental conditions we applied. Consequently, resonant ionization via these two lines (indicated by black arrows in Fig. 1) specifically target either camphor or fenchone in the mixture. Corresponding photoelectrons carry exclusively the information from the target molecule and can be used to infer the chirality by calculating the LPECD.

We demonstrate the feasibility of this approach by ionizing systematically all possible enantiomer mixtures of the structural isomers camphor and fenchone $\left(152 \mathrm{~g} \mathrm{~mol}^{-1}\right)$. For each mixture, we position the laser wavelength on either the $0_{0}^{0}$ transition of fenchone $(416.6 \mathrm{~nm})$ or camphor $(408.8 \mathrm{~nm})$ and

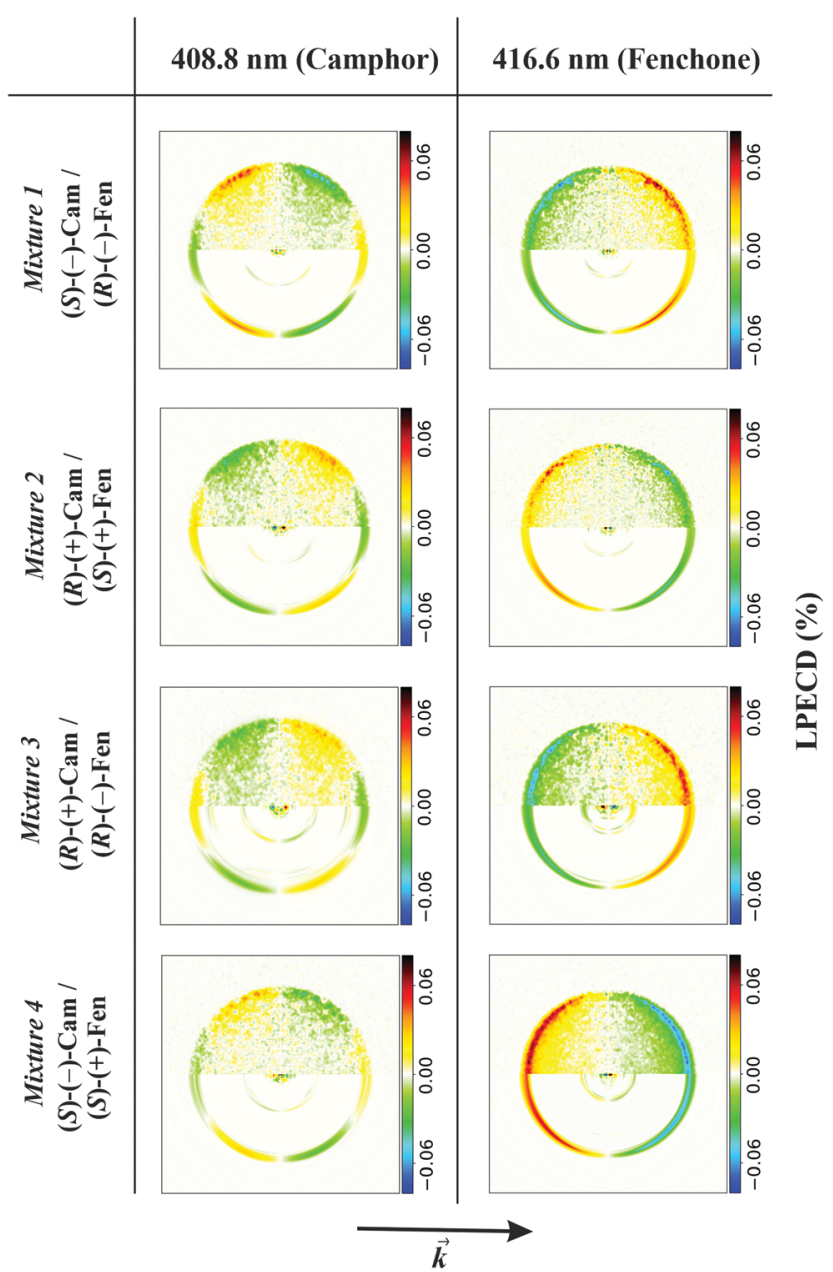

Fig. 2 Antisymmetrized PECD images obtained when ionizing fenchone/ camphor mixtures at $408.8 \mathrm{~nm}$ and $416.6 \mathrm{~nm}$. Experimental conditions were chosen like in the black spectrum of Fig. 1. In the upper half of each image, we show antisymmetrized raw data and in the lower half the Abelinverted image. The PECD images in the first column belong to ionized camphor and differ significantly from the images in the second column, which belong to ionized fenchone. Most obvious in the PECD images of camphor is the additional node described by the $c_{3}$ coefficient. The arrow at the bottom shows the direction of the laser beam. record corresponding PADs. In Fig. 2, we show typical antisymmetrized PECD images of the 4 mixtures.

It is obvious from Fig. 2 that PECD images recorded at $416.6 \mathrm{~nm}$ significantly differ from images recorded at $408.8 \mathrm{~nm}$. Most evident is the additional node in the PECD images at $408.8 \mathrm{~nm}$, which is typical for camphor. ${ }^{20}$ The PECD images change sign if one component in the mixture is substituted by the other enantiomer. This is independent of the other component in the mixture. From the images, we calculate LPECD values as explained above. We obtain values for $(R)-(-)$-fenchone of $15 \%$ (mixture 1 ) and $12 \%$ (mixture 3 ), for $(S)$ $(+)$-fenchone of $-12 \%$ (mixture 2 ) and $-14 \%$ (mixture 4 ), for $(S)-(-)$-camphor of $-9 \%$ (mixture 1 ) and $-6 \%$ (mixture 4 ), and for $(R)-(+)$-camphor of $5 \%$ (mixture 2 ) and $4 \%$ (mixture 3 ). The absolute uncertainty of each LPECD value is $\pm 3 \%$. The LPECD is not corrected for initial enantiomeric excess. Most values of fenchone are within the error of the experiment identical to the LPECD of pure fenchone as measured before. ${ }^{23}$ For camphor, no measurements of nanosecond PECD exist. We therefore performed reference experiments on pure $(S)$-(-)-camphor $(-4 \% \pm 2 \%)$ and $(R)-(+)$-camphor $(6 \% \pm 2 \%)$. The scatter of calculated values originates from limited available data. We intend to increase the data set in future experiments, which will allow analysis with better enantiomeric excess resolution.

In summary, we present a new technique to measure molecular chirality in mixtures using nanosecond high-resolution pulsed lasers. When ionizing the mixture via molecule-specific transitions, we exclusively record photoelectrons of the target molecule independent of other compounds in the mixture. This approach does not rely on the molecular mass and requires only little number density and ionization volume of molecules. It provides therefore a useful alternative to other techniques if suitable resonant ionization schemes are available.

\section{Conflicts of interest}

There are no conflicts to declare.

\section{Acknowledgements}

S. T. R., G. B. P. and T. S. acknowledge financial support within the LaserLab Europe network (Grant Agreement No. 871124). T. S. acknowledges support by the DFG under grant INST186/ 1302-1 and SCHA 1946/5-1. S. T. R., A. S., and T. B. acknowledge funding by the Deutsche Forschungsgemeinschaft (DFG, German Research Foundation) - Projektnummer 328961117 - SFB 1319 ELCH. Open access funding provided by the Max Planck Society.

\section{References}

1 In Gas Chromatography and Mass Spectrometry, ed. O. D. Sparkman, Z. E. Penton and F. G. Kitson, Academic Press, Amsterdam, 2nd edn, 2006.

2 H. G. Breunig, G. Urbasch, P. Horsch, J. Cordes, U. Koert and K.-M. Weitzel, ChemPhysChem, 2009, 10, 1199-1202. 
3 U. Boesl, A. Bornschlegl, C. Logé and K. Titze, Anal. Bioanal. Chem., 2013, 405, 6913-6924.

4 T. Ring, C. Witte, S. Vasudevan, S. Das, S. T. Ranecky, H. Lee, N. Ladda, A. Senftleben, H. Braun and T. Baumert, Rev. Sci. Instrum., 2021, 92, 033001.

5 P. Werkhoff, S. Brennecke, W. Bretschneider, M. Güntert, R. Hopp and H. Surburg, Z. Lebensm.-Unters. Forsch., 1993, 196, 307-328.

6 C. Bicchi, A. DAmato and P. Rubiolo, J. Chromatogr. A, 1999, 843, 99-121.

7 P. J. Marriott, R. Shellie and C. Cornwell, J. Chromatogr. A, 2001, 936, 1-22.

8 L. Wu and F. G. Vogt, J. Pharm. Biomed. Anal., 2012, 69, 133-147.

9 D. Patterson, M. Schnell and J. M. Doyle, Nature, 2013, 7450, 475-477.

10 D. Patterson and J. M. Doyle, Phys. Rev. Lett., 2013, 111, 023008.

11 S. R. Domingos, C. Pérez and M. Schnell, Annu. Rev. Phys. Chem., 2018, 69, 499-519.

12 B. Ritchie, Phys. Rev. A: At., Mol., Opt. Phys., 1976, 13, 1411-1415.

13 N. Böwering, T. Lischke, B. Schmidtke, N. Müller, T. Khalil and U. Heinzmann, Phys. Rev. Lett., 2001, 86, 1187-1190.

14 G. A. Garcia, L. Nahon, S. Daly and I. Powis, Nat. Commun., 2013, 4, 2132.

15 I. Powis, Advances in Chemical Physics, John Wiley and Sons, Ltd, 2008, ch. 5, pp. 267-329.

16 A. Zehnacker, Chiral Recognition in the Gas Phase, CRC Press, 2010.

17 L. Nahon, G. A. Garcia and I. Powis, J. Electron Spectrosc. Relat. Phenom., 2015, 204, 322-334.

18 S. Turchini, J. Phys.: Condens. Matter, 2017, 29, 503001.

19 C. Lux, M. Wollenhaupt, T. Bolze, Q. Liang, J. Köhler, C. Sarpe and T. Baumert, Angew. Chem., Int. Ed., 2012, 51, 5001-5005.

20 C. Lux, M. Wollenhaupt, C. Sarpe and T. Baumert, ChemPhysChem, 2015, 16, 115-137.

21 C. S. Lehmann, N. B. Ram, I. Powis and M. H. M. Janssen, J. Chem. Phys., 2013, 139, 234307.

22 A. Kastner, T. Ring, H. Braun, A. Senftleben and T. Baumert, ChemPhysChem, 2019, 20, 1416-1419.

23 A. Kastner, G. Koumarianou, P. Glodic, P. C. Samartzis, N. Ladda, S. T. Ranecky, T. Ring, S. Vasudevan, C. Witte, H. Braun, H.-G. Lee, A. Senftleben, R. Berger, G. B. Park, T. Schäfer and T. Baumert, Phys. Chem. Chem. Phys., 2020, 22, 7404-7411.
24 P. Krüger and K.-M. Weitzel, Angew. Chem., Int. Ed., 2021, 60, 17861-17865.

25 D. P. Singh, J. O. F. Thompson, K. L. Reid and I. Powis, J. Phys. Chem. Lett., 2021, 12, 11438-11443.

26 A. Kastner, T. Ring, B. C. Krüger, G. B. Park, T. Schäfer, A. Senftleben and T. Baumert, J. Chem. Phys., 2017, 147, 013926.

27 M. M. Rafiee Fanood, M. H. M. Janssen and I. Powis, J. Chem. Phys., 2016, 145, 124320.

28 S. Beaulieu, A. Ferré, R. Géneaux, R. Canonge, D. Descamps, B. Fabre, N. Fedorov, F. Légaré, S. Petit, T. Ruchon, V. Blanchet, Y. Mairesse and B. Pons, New J. Phys., 2016, 18, 102002.

29 G. Nalin, K. Fehre, F. Trinter, N. M. Novikovskiy, N. Anders, D. Trabert, S. Grundmann, M. Kircher, A. Khan, R. Tomar, M. Hofmann, M. Waitz, I. Vela-Pérez, G. Kastirke, J. Siebert, D. Tsitsonis, H. Fukuzawa, K. Ueda, J. B. Williams, D. Kargin, M. Maurer, C. Küstner-Wetekam, L. Marder, J. Viehmann, A. Knie, T. Jahnke, M. Ilchen, R. Dörner, R. Pietschnig, P. V. Demekhin and M. S. Schöffler, Phys. Chem. Chem. Phys., 2021, 23, 17248-17258.

30 A. Kastner, C. Lux, T. Ring, S. Züllighoven, C. Sarpe, A. Senftleben and T. Baumert, ChemPhysChem, 2016, 17, 1119-1122.

31 L. Nahon, L. Nag, G. A. Garcia, I. Myrgorodska, U. Meierhenrich, S. Beaulieu, V. Wanie, V. Blanchet, R. Géneaux and I. Powis, Phys. Chem. Chem. Phys., 2016, 18, 12696-12706.

32 M. M. Rafiee Fanood, N. Bhargava Ram, C. S. Lehmann, I. Powis and M. H. M. Janssen, Nat. Commun., 2015, 6, 7511.

33 M. M. Rafiee Fanood, I. Powis and M. H. M. Janssen, J. Phys. Chem. A, 2014, 118, 11541-11546.

34 A. Comby, C. M. M. Bond, D. Descamps, J. Miles, S. Petit, S. Rozen, J. B. Greenwood, V. Blanchet and Y. Mairesse, Nat. Commun., 2018, 9, 5212.

35 G. Westphal, M. Wallrabe and T. Schäfer, J. Phys. Chem. C, 2020, 124, 799-804.

36 G. Westphal, J. Wega, R. E. A. Dissanayake and T. Schäfer, J. Chem. Phys., 2020, 153, 054707.

37 G. B. Park, B. C. Krüger, S. Meyer, D. Schwarzer and T. Schäfer, J. Chem. Phys., 2016, 144, 194308.

38 V. Papadakis and T. N. Kitsopoulos, Rev. Sci. Instrum., 2006, 77, 083101.

39 G. A. Garcia, L. Nahon and I. Powis, Rev. Sci. Instrum., 2004, 75, 4989-4996. 